\title{
Thrombectomy approach for access maintenance in the end stage renal disease population: a narrative review
}

\author{
Vincent $\mathrm{Wu}^{1}$, Sanjeeva P. Kalva ${ }^{1}$, Jie Cui ${ }^{2}$ \\ ${ }^{1}$ Division of Interventional Radiology, Department of Radiology, Massachusetts General Hospital/Harvard Medical School, Boston, MA, USA; \\ ${ }^{2}$ Nephrology Division, Department of Medicine, Massachusetts General Hospital/Harvard Medical School, Boston, MA, USA \\ Contributions: (I) Conception and design: All authors; (II) Administrative support: All authors; (III) Provision of study materials or patients: All \\ authors; (IV) Collection and assembly of data: None; (V) Data analysis and interpretation: None; (VI) Manuscript writing: All authors; (VII) Final \\ approval of manuscript: All authors. \\ Correspondence to: Vincent Wu, MD. Division of Interventional Radiology, Department of Radiology, Massachusetts General Hospital/Harvard \\ Medical School, Boston, MA 02114, USA. Email: vwu3@mgh.harvard.edu.
}

Objective: This article reviews current practices and outcomes in endovascular thrombectomy techniques for the treatment of thrombosed arteriovenous grafts (AVGs) and fistulas (AVFs).

Background: Arteriovenous (AV) access allows patients with end-stage renal disease (ESRD) to receive hemodialysis. Thrombosis of AV access can lead to delay in hemodialysis or abandonment of access requiring dialysis catheter placement. Endovascular approach has become the preferred treatment option for thrombosed access over surgery. Interventions include removal of thrombus from the AV circuit and treatment of the underlying anatomical abnormality, such as an anastomotic stenosis. Thrombolysis, or the act of dissolving thrombus, is performed by using infusion catheters or pulse injector devices for the administration of fibrinolytic agents. Thrombectomy, or the mechanical removal of thrombus, is performed by using embolectomy balloon catheters, rotating baskets or wires, rheolytic and aspiration mechanisms. Adjunctive methods such as cutting balloon angioplasty, drug-coated balloon (DCB) angioplasty, and stent placement are also used to treat stenoses in the AV circuit. Complications of these procedures include vessel rupture, arterial embolism, pulmonary embolism (PE), and paradoxical embolism to the brain.

Methods: This narrative review article was written based on literature search from electronic databases, including PubMed and Google Scholar.

Conclusions: The understanding of thrombectomy techniques and their potential complications is essential in the management of patients with thrombosed AV access.

Keywords: Thrombectomy; thrombolysis; declot; arteriovenous fistula (AVF); arteriovenous graft (AVG)

Submitted Aug 20, 2021. Accepted for publication Dec 15, 2021. Published online Dec 28, 2021.

doi: $10.21037 / \mathrm{cdt}-21-523$

View this article at: https://dx.doi.org/10.21037/cdt-21-523

\section{Introduction}

The prevalence of chronic kidney disease (CKD) is increasing worldwide and contributing to the global burden of disease. Hemodialysis is the most common renal replacement modality for patients with end-stage renal disease (ESRD). Compared to central venous catheters, arteriovenous fistula (AVF) and graft (AVG) are the preferred access for chronic hemodialysis treatment due to lower hospitalization risk and mortality $(1,2)$. Poorer outcomes in catheter-dependent patients are associated with increased risk of sepsis and bacteremia (2). However, long-term vascular accesses typically require frequent interventions to maintain access patency, which is not only a significant burden on health care resources, but also impacts patients' quality of life with implications on morbidity and mortality. 
Thrombosis is the leading cause of access loss in AVF and AVG, accounting for $65-85 \%$ of the cases (3). Thrombosis rate for AVF is 0.24 events per 1,000 patient days (4), and AVG thrombosis occurs 0.2 events per patient during 2 years follow-up (5). Access thrombosis is commonly caused by underlying venous or arterial anastomotic stenoses. Systemic factors that can increase the risk of access thrombosis include hypercoagulability $(6,7)$, low ejection fraction (8), hypotension and hemoconcentration (9). Cannulation complications resulting in hematomas can also lead to access thrombosis (10).

Thrombosed access leads to hemodialysis treatment delays, treatment omissions, inpatient admissions and abandonment of access requiring dialysis catheter placement (3). Thrombus formed in arteriovenous (AV) access is comprised of two components: soft, friable clot that disintegrates easily in the venous outflow and a firm fibrin plug near the anastomosis (11). To date, no FDA-approved pharmacological therapies can prevent access thrombosis. The long-term effect of anti-platelet therapy and warfarin on access patency remains unclear $(12,13)$. Thrombosed access should be treated as soon as possible to avoid delay on dialysis treatment and catheter placement. When fresh thrombus hardens into adherent thrombus in the native vein of an AVF, thrombectomy becomes less likely to be successful (14). As a result, thrombectomy in AVF should be performed within days, whereas thrombectomy in AVG can still be successful up to a week. Early experiences with thrombosed $\mathrm{AV}$ access required surgical thrombectomy, which usually requires hospitalization and operation room resources. However, with the development of intravascular techniques, endovascular thrombectomy has become the standard of care for thrombosed access. Under imaging guidance, not only can the thrombus be removed, underlying anatomical abnormalities (such as stenoses) can also be treated. As reported by prior studies, procedure success rates for thrombosed fistula or graft by endovascular approach have a range of $70-90 \%(15-18)$. Compared to 20 years ago, the number of endovascular access thrombectomy procedure has doubled for AVF and tripled for AVG (19). This article aims to review current practices, outcomes, and potential complications in endovascular thrombectomy techniques for the treatment of thrombosed AVGs and AVFs. This review article was written based on literature search of electronic databases, including PubMed and Google Scholar. We present the following article in accordance with the Narrative Review reporting checklist (available at https://cdt. amegroups.com/article/view/10.21037/cdt-21-523/rc).

\section{Access surveillance and physical exam}

The concept of vascular access surveillance is early detection of access thrombosis, which can lead to earlier intervention to prevent thrombotic events and minimizes the number of missed dialysis sessions. Most thrombosed accesses have underlying stenoses. Over the years, there has been development of noninvasive methods to detect access stenoses so these can be intervened before thrombosis occurs. These tests include static dialysis venous pressure, flow monitoring, ultrasound and physical examination. However, the utility of dialysis access surveillance in predicting the risk of a thrombotic event remains controversial. A recent randomized study (5) showed that monthly access blood flow monitoring and ultrasound dilute technique can decrease thrombotic events from 0.22 event per person to 0.11 event per person compared to monthly physical examination. However, patients with prior access thrombosis were excluded from this study. Other studies have raised the concern that surveillance may increase the number of unnecessary angioplasties $(20,21)$. The information derived from access surveillance is limited because the $\mathrm{AV}$ circuit is dynamic, and many factors can affect the pressure and flow of the circuit, include cannulation technique, needle placement, hemodynamics during dialysis, and the number and location of stenotic segments. In general, indications for endovascular evaluation include access with a history of difficult cannulations, excessive bleeding following removal of needles, high arterial or venous pressures, low access flow, and significant recirculation.

On physical examination, a thrombosed access would manifest as a lack of thrill and bruit (22). Access may be pulsatile if the $\mathrm{AV}$ anastomosis is patent but the venous outflow is occluded. In addition, an erythematous and tender fistula is suggestive of inflammatory thrombophlebitis. Ultrasound can be a useful tool in confirming the presence and location of thrombus. The findings include a lack of flow on pulse-wave doppler and non-compressibility of the vein or graft. Echogenicity of intraluminal material would depend on the chronicity of the clot; the more chronic the clot the more echogenic it becomes.

\section{Patient evaluation and contraindications to endovascular thrombectomy}

Evaluation for endovascular thrombectomy of clotted AV access should include prior imaging (e.g., fistulograms, 

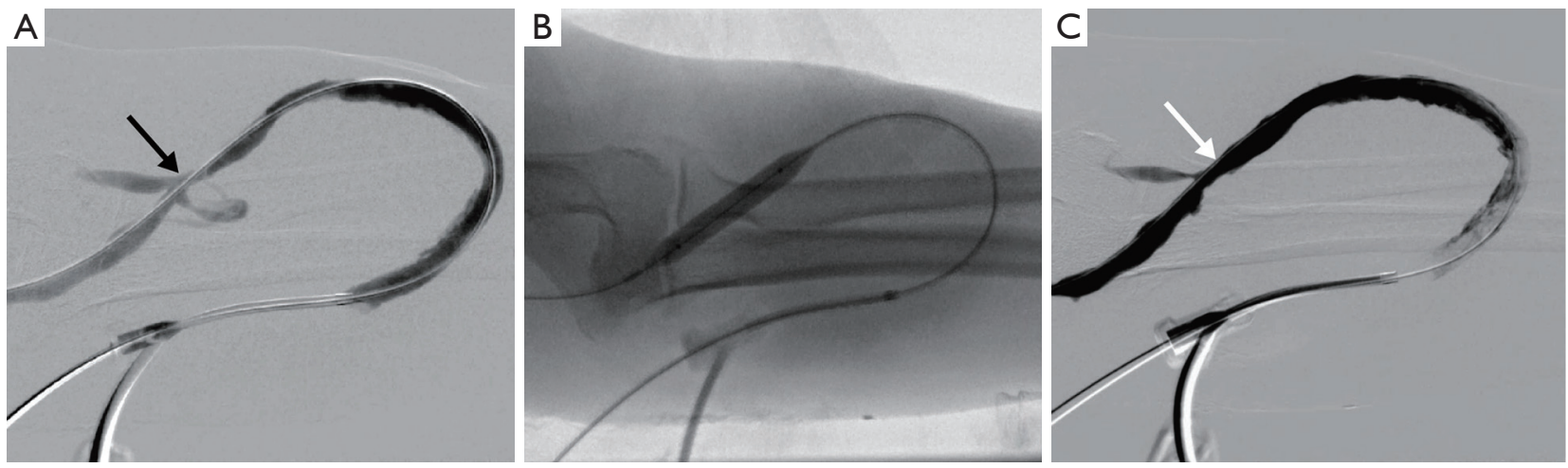

Figure 1 AVG venous anastomotic stenosis. (A) Graftogram through the antegrade vascular sheath during thrombectomy of a clotted forearm loop graft shows severe stenosis at the venous anastomosis (black arrow). This finding was likely the underlying cause of the graft thrombosis. (B) Angioplasty of the venous anastomotic stenosis. (C) Post-angioplasty graftogram demonstrates significant luminal gain with less than $30 \%$ residual stenosis at the venous anastomosis (white arrow). AVG, arteriovenous graft.

graftograms, ultrasound), prior interventions (e.g., thrombectomy, angioplasty), cardiopulmonary status, last hemodialysis session, laboratory values (e.g., potassium), coagulopathy (e.g., anticoagulation or antiplatelet therapy), medications and allergies (e.g., heparin, contrast). When consenting for the procedure, patients should be informed of the possibility of hemodialysis catheter placement in the event of unsuccessful thrombectomy.

Relative contraindications for endovascular AV access thrombectomy include: (I) poor pulmonary or cardiac reserve; (II) contraindication for tissue plasminogen activator (tPA); (III) active hemorrhage; (IV) hemodynamic instability and severe hypotension; (V) mega fistulas; (VI) intracardiac right-to-left shunt; (VII) hyperkalemia requiring urgent hemodialysis; (VIII) AV access infection; (IX) sepsis/bacteremia; and (X) recently created anastomosis.

\section{Endovascular thrombectomy techniques and outcomes}

Treatment of AV access thrombosis using endovascular techniques has been considered a viable alternative to surgical thrombectomy. Endovascular approach is the mainstay in modern times due to their minimally invasive nature (23). The availability of a wide selection of thrombectomy devices also popularized the endovascular approach. Additional benefits of endovascular approach include preserving the existing access without reducing sites for future access (24), minimal delays in dialysis, repeatability of procedure, and the ability to perform angioplasty and thrombectomy in the same setting (25). The choice of thrombectomy technique depends on primarily on operator preference, patient specific conditions, device cost, and device availability.

\section{Fundamental principals and techniques}

In general, $\mathrm{AV}$ access thrombectomy encompasses two main steps: (I) removal of clot and (II) treatment of underlying significant stenosis in the circuit that precipitated the thrombosis. Procedural steps may not always adhere to this order. Anatomically significant stenosis is defined as narrowing $>50 \%$ of normal vessel diameter (25). Stenosis may occur in various locations in the $\mathrm{AV}$ circuit, such as the central veins, venous outflow, juxta-anastomotic segment, or AV anastomosis. Additionally, the process of declotting may include "thrombectomy" versus "thrombolysis". Thrombectomy refers to mechanically removing clot by means of, for example, aspiration or Fogarty balloon sweeps. On the other hand, thrombolysis involves dissolving clot by means of instillation of pharmacological drugs such as tPA in the thrombus. Both techniques can be utilized either alone or in combination in a standard thrombectomy case.

Experiences can differ between treating AVG and AVF thrombosis. AVG thrombectomy tends to be less challenging due to uniform size of the graft, smaller clot burden, and predictable anatomy with sites of stenosis that are typically located near the venous anastomosis (Figure 1). On the other hand, AVF thrombectomy may be more difficult to perform for several reasons $(26,27)$. First, the presence of aneurysms of the native vein can increase clot burden and 
make it more difficult to catheterize. Second, the presence of venous collaterals can make it more challenging to navigate the anatomy. Third, the location of culprit stenosis is more variable. Lastly, in a newly created AVF, the vessel diameter can be smaller, resulting in difficulty in cannulation, vessel occlusion with vascular sheaths, and increased risk of vessel rupture with angioplasty.

$\mathrm{AV}$ access thrombectomy can be performed under conscious sedation with intravenous opioids (e.g., fentanyl) and benzodiazepine (e.g., midazolam) in the angiographic suite. In patients with severe cardiopulmonary dysfunction or other co-morbidities, anesthesia support may be needed. Local anesthetics (e.g., 1\% lidocaine) are given at the access sites. Systemic intravenous heparin is given at a dose of 50-60 units per kilogram in the beginning of the case followed by 1,000 units every hour. In patients with heparin allergy or heparin-induced thrombocytopenia, intravenous argatroban or bivalirudin can be used during the procedure.

\section{Thrombolysis}

Endovascular declotting of dialysis access dates back to 1984 when Hunter et al. (28) showed that thrombosed fistulas can be restored using streptokinase infusion percutaneously. Another paper from 1985 showed a $77 \%$ success rate in declotting thrombosed $\mathrm{AV}$ access using endovascular thrombolysis with minimal complications (29). Acutely formed thrombus is responsive to fibrinolytic agents such as streptokinase, urokinase, and tPA. Recombinant tPA now includes alteplase, reteplase, and tenecteplase. Several studies in the 1990's demonstrated that infusion thrombolysis for thrombosed AVG had a technical success rate of $60-80 \%$ with 6 -month patency rate ranging from $13 \%$ to $80 \%$ (30-33). Authors of these studies observed that patency rates were improved when AVG stenoses were corrected immediately by angioplasty or surgery. Infusion catheters were either introduced into the clotted access as a single catheter or using a crossed catheter technique, where two punctures were made on two ends of the graft and catheters were inserted from either end with catheter tips placed at the arterial and venous end (33). Catheter infusion of fibrinolytic agents would last for 3-24 hours (34). Several groups at the time also described methods to enhance the effect of thrombolysis, including the use of rotating angled or pigtail catheters for clot maceration $(35,36)$ or manual graft massage (37).

The lyse-and-wait technique was described in 1997 by Cynamon et al. (38) where thrombolytic agents were administered into the clotted AVG through a catheter in the pre-procedure area, several hours before the patient was brought into the angiographic suite. The goal was to reduce most of the clot burden prior to the main procedure. In the angiography suite, the operator could then focus on addressing the underlying cause of thrombosis such as angioplasty of culprit stenosis or removal of arterial plug.

\section{Pulsed-spray thrombolysis}

Pulsed-spray thrombolysis was introduced where a pulse injector was used to instill high pressure pulses of highly concentrated fibrinolytic agents into clot through multisidehole catheters $(39,40)$. Valji et al. (41) described the use of $25,000 \mathrm{IU} / \mathrm{mL}$ of urokinase admixed with heparin that was instilled directly into the clot using a high-pressure pulse spray for 15-20 minutes. Technical success rate was $96 \%$ and the total procedure time was 67 minutes. Several other groups also demonstrated feasibility of the pulsedspray thrombolysis technique, where technical success rates were $85-94 \%$ with approximately 1 hour of lysis time $(33,42)$. The shorter lysis time was an advantage compared to conventional thrombolysis methods where patients required overnight infusions under intensive care unit monitoring. Cooper demonstrated technical and clinical success rate of $94 \%$ in treating clotted AVG using the Pulse Spray Injector system (AngioDynamics, Glens Falls, NY, USA) by delivering $2 \mathrm{mg}$ of tPA through a multisidehole infusion catheter (43). The same study described thrombolysis times of 10-16 minutes with primary patency rates of $71 \%$ at 30 days and $47 \%$ at 90 days using this technique with minimal complications.

\section{Pharmaco-mechanical thrombolysis (PMT)}

PMT technique is the most common thrombectomy technique that utilizes balloon catheters and tPA with or without dedicated advanced thrombectomy devices. A study utilizing PMT technique for AVG thrombectomy (44) showed that technical and clinical success rates of $95 \%$. This study also reported that the primary patency rate of AVG after PMT was $45 \%$ and $22 \%$ at 12 and 24 months, respectively, without major complications.

We typically begin with antegrade access of the AVF or AVG using a micropuncture access system under ultrasound guidance, which is upsized to a 6 or 7 French short vascular sheath. The size of the vascular sheath depends on which devices are used, including the angioplasty balloon size. A 
5 French angled catheter and $0.035^{\prime \prime}$ hydrophilic guidewire (Terumo Medical Co., Somerset, NJ, USA) are used to navigate across the clotted venous outflow into the central veins. A venogram of the central veins is performed to evaluate for any central venous stenosis involving superior vena cava, brachiocephalic and subclavian veins. A "pullback" venogram is performed by injecting the contrast material through the catheter as it is retracted from the central veins to the body of the AVF or AVG. This would reveal the thrombus burden of venous outflow as well as any significant venous stenosis. We routine administer up to $4 \mathrm{mg}$ of tPA (e.g., alteplase) within the thrombus for thrombolysis, unless there are relevant contraindications. In addition, we administer intravenous heparin at 50-60 units/ $\mathrm{kg}$ body weight. We also prefer to perform balloon maceration of clot in the venous outflow early to create a channel for blood flow. Prior literature has recommended that venous outflow clot should be removed first to minimize the risk of clot fragments embolizing into the forearm artery (27).

Next, retrograde access is obtained using a micropuncture access system and then upsized to a 5 or 6 French short vascular sheath. A 5 French angled catheter and 0.035" hydrophilic guidewire are used to navigate through the body of the AVF or AVG across the arterial anastomosis into the upstream artery. Injection of contrast material in the artery would be performed to evaluate the extent of arterial thrombus as well as downstream artery patency. An over-the-wire Fogarty thrombectomy catheter (Edwards Lifesciences, Irvine, CA, USA) is then introduced through the sheath and used to perform sweeps to remove the arterial plug to open the arterial inflow. This maneuver should be performed gently and carefully to avoid thrombi dislodgement into the arterial system. An arterial plug represents thrombi located at the AV anastomosis that is made of fibrin, red blood cells, and platelets (45). Pulsatility of the AVF or AVG on physical exam is an indication that inflow patency has been restored.

If present, stenosis near the arterial anastomosis should be treated with balloon angioplasty. Subsequently, balloon maceration is performed throughout the extent of the clot. Once flow is established after balloon maceration, manual aspiration through the side-ports of the vascular sheaths can also be attempted to remove the clot. This thromboaspiration technique has been described in a series of 520 cases of thrombosed AVF and confers a high clinical success rate of $91.1 \%$ (46). Once clot burden has been reduced, additional angioplasty of hemodynamically significant stenoses should be performed with appropriately sized balloons. Declot is complete once satisfactory antegrade blood flow is established on completion angiograms and there is restoration of thrill on physical exam (Figure 2). Vascular sheaths are then removed after hemostasis is achieved with pursestring sutures (47). These pursestring sutures are removed after 1 hour while patient is in the recovery room.

\section{Mechanical thrombectomy}

A wide selection of thrombectomy devices is now available for the treatment of clotted AV access. Arrow-Trerotola Percutaneous Thrombolytic Device (PTD) (Teleflex; Wayne, PA, USA) is a spinning nitinol basket designed for clot maceration of thrombosed AV access. The device comes attached to a hand-held rotator drive unit and introducer sheath. The nitinol basket conforms to the vessel wall to maximize contact with clot during use. Rocek et al. (48) showed that using Arrow-Trerotola PTD in declotting native AVF had technical and clinical success rates of $100 \%$ and $90 \%$, respectively, with a 6-month primary patency rate of $60 \%$. Several studies were done to compare the device to more traditional thrombectomy techniques. Trerotola et al. (49) compared the Arrow-Trerotola PTD to pulsedspray thrombolysis and showed that success rates and immediate patency rates were similar between the two techniques in treating clotted AVG. Arrow-Trerotola PTD was associated with shorter procedure times in this study. Additionally, when comparing Arrow-Trerotola PTD to the lyse-and-wait technique with urokinase in clotted AVG (50), similar immediate success rates and average patency time were observed between the two techniques. However, the lyse and wait technique was associated with lower cost.

CLEANER Rotational Thrombectomy System (Argon Medical; Plano, TX, USA) is a rotating sinusoidal wire attached to a battery powered hand-held controller that is designed for clot maceration in thrombosed vessels. It is FDA approved for the treatment of deep vein thrombosis, but the device is known to be used in clotted AV access as well (Figure 3). The width of the sinusoidal wire conforms to the vessel diameter that maximizes contact with clot. It has a built-in sideport can be used for administration of thrombolytics. Recently, a group in Singapore showed that using the CLEANER device in conjunction with thrombolytics in treating clotted AVG and AVF, they were able to achieve technical and clinical success rates of $88 \%$ resulting in $65 \% 1$-month and $47 \% 3$-month primary patency rates (51). The rotational thrombectomy system 

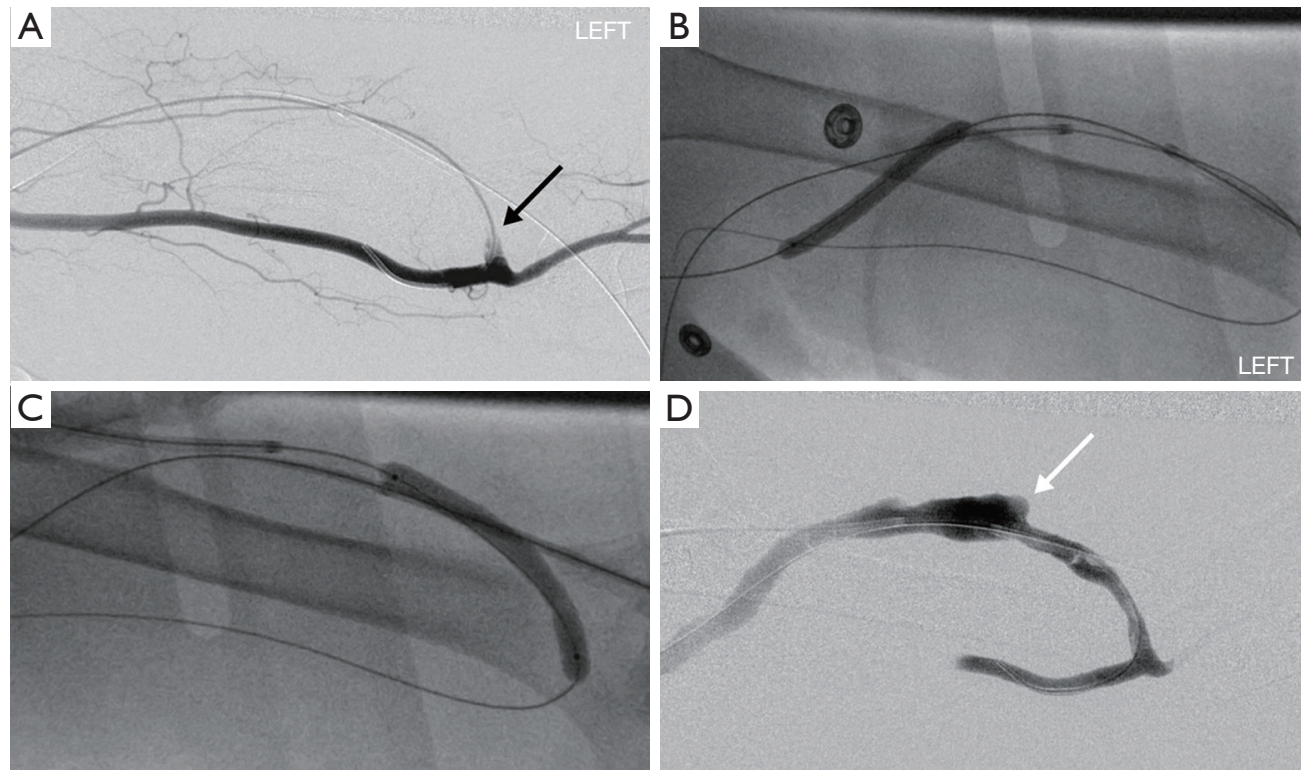

Figure 2 Pharmaco-mechanical thrombectomy of clotted brachiocephalic AVF. (A) Fistulogram with retrograde diagnostic catheter in the upstream brachial artery shows AVF thrombosis and occlusion near the AV anastomosis (black arrow). (B) Balloon maceration of venous outflow clot via the antegrade access. (C) Balloon maceration of juxta-anastomotic segment clot via the retrograde access. (D) Postthrombectomy fistulogram shows improved opacification of the AVF and antegrade flow. Venous aneurysms can be seen with native fistulas (white arrow). AVF, arteriovenous fistula; AV, arteriovenous.
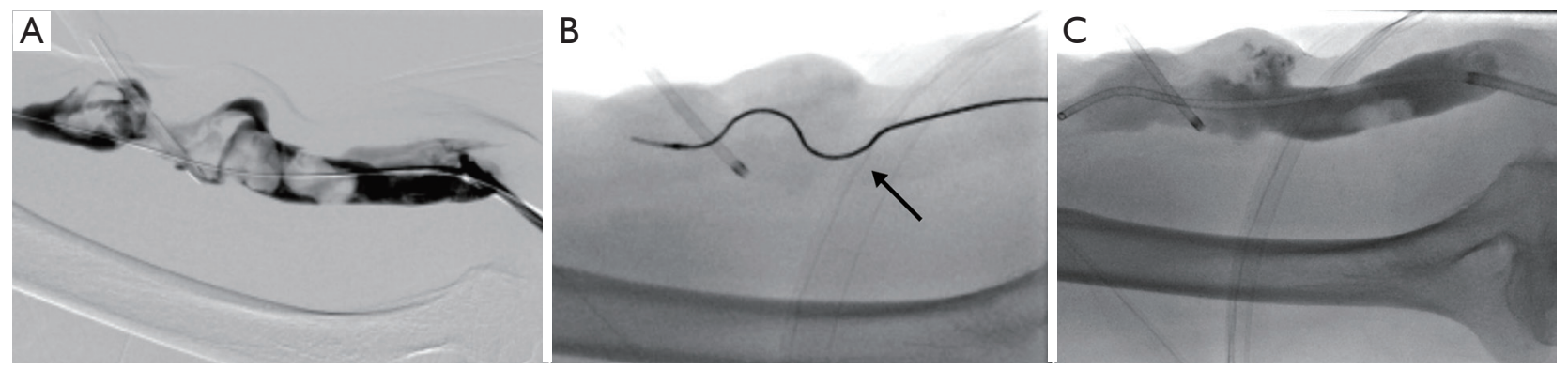

Figure 3 CLEANER Rotational Thrombectomy System (Argon Medical). (A) Brachiocephalic fistulogram shows multiple large thrombi within multifocal venous aneurysms. (B) Clot maceration using the rotating sinusoidal wire from the CLEANER device (black arrow). (C) Post-thrombectomy fistulogram shows decreased clot burden with residual thrombi in the aneurysmal segments.

can be successfully used to treat patients with aneurysmal AVF. Other groups have shown that the CLEANER device can be a safe and effective bail-out option in restoring patency to thrombosed $\mathrm{AV}$ shunts not responding to balloon thrombectomy methods (52). One technical caveat is that the CLEANER device does not advance over a wire. We recommend placing the vascular sheath tip near the treatment site before introducing the CLEANER device. Since the guidewire may need to be removed to allow the introduction of the CLEANER device, there is the risk of losing access especially in the presence of severe venous stenosis or total venous occlusion. Although mechanical thrombectomy devices have been reported to not cause damage to the endothelial wall, careful patient selection is still advised.

\section{Rheolytic thrombectomy}

Flow-based thrombectomy systems utilize a high-velocity saline jet (created by a pump set and drive unit) to generate 
a negative pressure gradient (Venturi effect) at the catheter tip. This results in fragmentation of the thrombus near the catheter tip and evacuation of thrombus fragments through the in-flow port of the catheter into a collection bag. The AngioJet Peripheral Thrombectomy System (Boston Scientific; Marlborough, MA, USA) is a modern iteration of such device. Angiojet has a Power-Pulse Delivery mode where thrombolytics can be delivered prior to clot evacuation. Other variations of rheolytic thrombectomy devices have been described in the past and function in a similar manner, including the Amplatz thrombectomy device (ATD; Microvena, White Bear Lake, MN, USA) (53) and the Hydrolyser (Cordis; Miami, FL, USA) (54).

Angiojet combined with Fogarty balloon is commonly used for cases that are refractory to conventional balloon maceration and thrombectomy. In our practice, once wire access is achieved across the clotted segments of the AV circuit, we first advance the Angiojet catheter over the wire and deliver tPA (about $4 \mathrm{mg}$ ) into the clot using the Power-Pulse Delivery mode. After allowing the lytic agent to sit in clot for about 10-20 minutes, we then switch to Thrombectomy mode and begin evacuating the clot into the catheter. We typically make 3-4 passes with the Angiojet catheter across the clotted segments. Compared to CLEANER system, Angioject thrombectomy is performed over a wire, which has the advantage of preserving wire access. Note that Angiojet use is associated with transient bradyarrhythmias, which typically resolve after the device is turned off (55).

Several studies have investigated the use of Angiojet in AV access. Littler et al. (56) showed that Angiojet thrombectomy of AVG and AVF resulted in technical success rate of primary $91 \%$ with primary patency rates of $71 \%, 60 \%$, and $37 \%$ at 1,3 , and 6 months, respectively. Adverse events specific to Angiojet included transient chest tightness and dyspnea that may be related bradycardia caused by the hemolytic effect and the release of adenosine. PEARL I (PEripheral Use of AngioJet Rheolytic Thrombectomy with Mid-Length Catheters) Registry reported technical success rate of $88 \%$ using the device in clotted AV access. At 3-month follow-up, 53\% of AVG remained patent and $86 \%$ of AVF remained patent (57). Bermudez et al. (58) in 2017 also demonstrated similar thrombectomy success rates of clotted $\mathrm{AV}$ access using Angiojet with primary patency rates of $52 \%, 41 \%$, and $23 \%$ at 3, 6, and 12 months, respectively.

A recent comparison between Angiojet and surgical thrombectomy in clotted AV access did not show significant differences in success rates as well as primary and secondary patency rates at 12 months (59). Percutaneous technique was associated with decreased bleeding risk compared to its surgical counterpart in this study. In contrast, Aydin et al. (60) demonstrated that the use of Angiojet PMT in native AVF had improved primary patency rates compared to surgical thrombectomy at 6 and 12 months.

The newer generation Angiojet catheters are available in different sizes. The 4 and 6 French catheters are typically used in peripheral arterial cases, whereas the 8 French (ZelanteDVT) catheter is designed for venous thrombectomy. In our experience, we observe increased technical success rates in $\mathrm{AV}$ access declot with the 8 French catheter. Note that the Angiojet AVX catheter is a 6 French catheter designed specifically for the treatment of AV access due to its shorter catheter length at $50 \mathrm{~cm}$. Other authors have experienced improved thrombectomy success rates with newer generation devices (56). An accessory called the ClotHunter was recently developed for Angiojet. The ClotHunter is attached to a helical wire that is inserted into the guidewire lumen of the ZelanteDVT catheter. A dial on the ClotHunter hand-held system creates a rotatory helical motion for the ZalenteDVT catheter during thrombectomy that broadens the treatment area within the vessel. Further studies are required to investigate the effectiveness of this accessory, especially in the $\mathrm{AV}$ access thrombosis cohort.

\section{Aspiration thrombectomy}

Indigo thrombectomy system (Penumbra; Alameda, CA, USA) is a vacuum-assisted device that enables continuous thrombus aspiration through end-hole catheters of various sizes. The CATD Indigo system is an 8 French suction catheter designed for $\mathrm{AV}$ access thrombectomy given its shorter length of $90 \mathrm{~cm}$. The newly released Lighting system is compatible with the CAT12 12 French catheter that is designed for improved venous clot retrieval. In order to minimize blood loss while using the large CAT12 catheter, the Lightning system has a sensor that modulates the suction power depending on blood flow.

In our practice, we prefer the use of aspiration thrombectomy with the Indigo system in patients who have contraindications to thrombolysis. Due to the advantage of clot removal, we also prefer the use of the Indigo system in patients with low pulmonary reserve who are at a greater risk of decompensation if pulmonary embolism (PE) develops from the declot procedure. Removal of clot should also be a priority in patients with right-to-left shunt to 

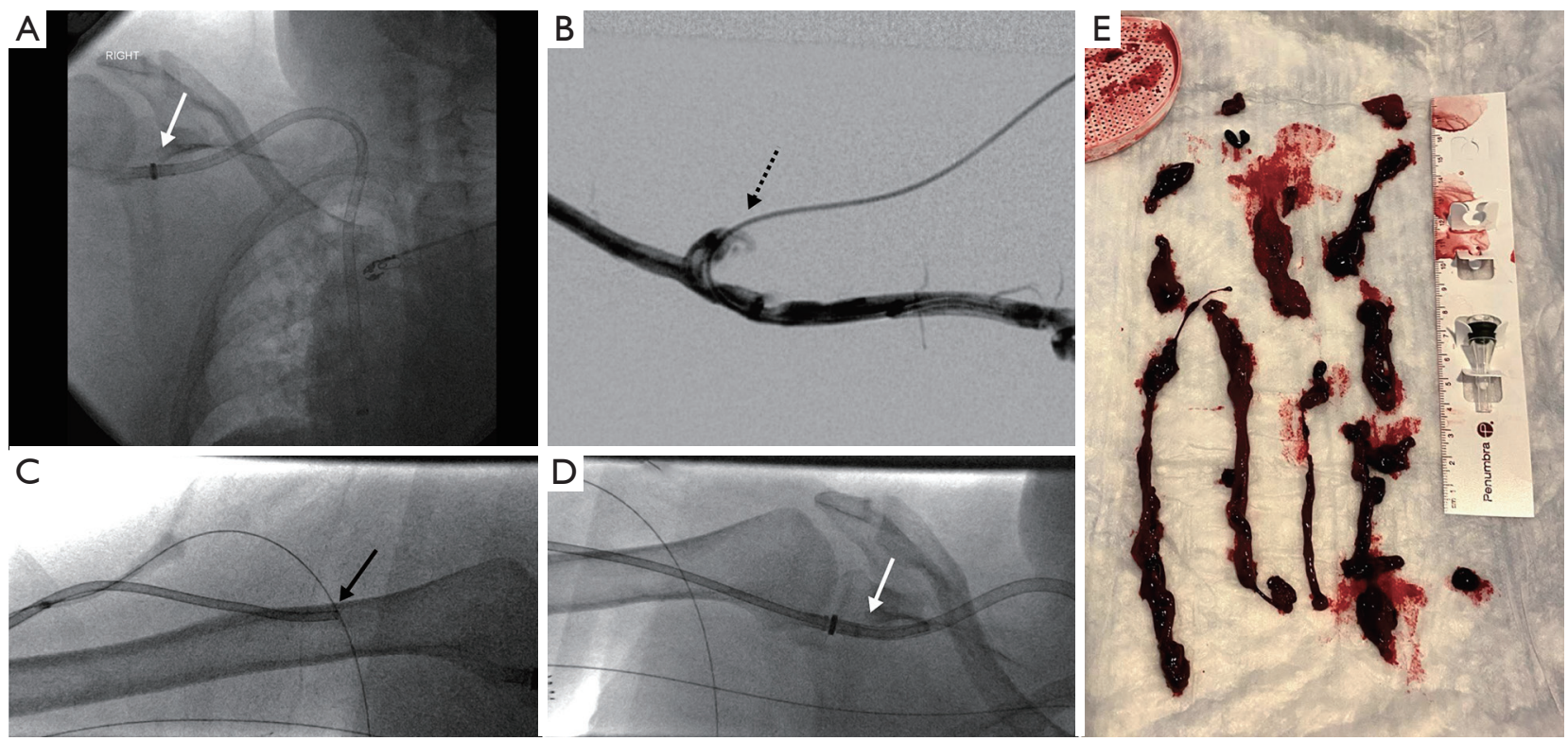

Figure 4 Aspiration thrombectomy of clotted HeRO graft using the Indigo system (Penumbra). (A) HeRO graft with venous outflow component extending into the upper right atrium (white arrow). (B) Graftogram with retrograde diagnostic catheter in the artery shows graft thrombosis and occlusion near the AV anastomosis (dashed black arrow). (C) CAT12 12 French aspiration catheter advanced into the arterial graft component of the HeRO graft (black arrow). (D) Aspiration catheter advanced into the venous outflow component of the HeRO graft (white arrow). (E) Clot extracted using the Indigo system. HeRO, Hemodialysis Reliable Outflow; AV, arteriovenous.

minimize the risk of paradoxical embolism (61). Moreover, thrombosed Hemodialysis Reliable Outflow (HeRO) grafts typically carry larger clot burden compared to conventional AVG and AVF. For this reason, we prefer aspiration thrombectomy in thrombosed HeRO grafts to minimize the risk of clinically significant PE (Figure 4).

Marcelin et al. (62) showed $97 \%$ technical success rate in the treatment of clotted AVG and AVF using the Indigo system with a 6-month primary patency rate of $71 \%$. The average procedure time was reported to be 38 minutes. No hemolytic complications were seen with the use of the Indigo system compared to Angiojet. Piacentino et al. (63) reported a clinical success rate of $80 \%$ in AVG clot removal in 10 patients using the Indigo system. They reported a much lower 6-month primary patency rate of $37.5 \%$ compared to other similar studies, which was likely because most of the grafts in this cohort already had numerous prior interventions.

\section{Adjunctive treatment}

$\mathrm{AV}$ access thrombosis occurs due to underlying venous outflow or inflow stenosis associated with downstream cellular events such as neointimal hyperplasia $(64,65)$ or upstream events such as surgical trauma, inflammation, and injury from vessel wall punctures (25). In particular, the most common cause of failure of AVG is venous anastomosis stenosis (66). These stenotic lesions are routinely treated with plain balloon angioplasty (POBA) using high pressure balloons to maintain patency of the AV circuit.

Drug-coated balloons (DCB) angioplasty has been shown in randomized-control-trials to be superior to POBA in the treatment of $\mathrm{AV}$ access stenosis in terms of long term patency and re-stenosis rates $(67,68)$. DCB uses paclitaxel and sirolimus that are known to inhibit neointimal hyperplasia. The Lutonix AV Clinical Trial initially showed that paclitaxel-coated balloon-assisted angioplasty had similar short term target lesion patency rates at 180 days compared to POBA (69), however long term patency rates with DCB were higher at 9 and 12 months (70). The IN.PACT AV Access Study showed that the Admiral paclitaxel-coated balloon angioplasty (Medtronic; Santa Rosa, CA, USA) was superior to POBA for the treatment of stenotic lesions in dysfunctional hemodialysis access at 6 months (71). In AVG, Liao et al. (72) reported modest improvement in primary patency of venous anastomotic 
stenosis with the use of DCB compared to POBA. However, accesses with prior thrombosis events were excluded from these studies. One recent study showed that the use of sirolimus DCB angioplasty at the graft vein junction after declot of thrombosed upper limb AVG resulted in primary patency rates of $76 \%$ and $65 \%$ at 3 and 6 months, respectively (73).

Cutting balloon angioplasty may be considered for stenotic lesions refractory to conventional high pressure balloon angioplasty. The use of cutting balloons may produce better patency rates in AVF stenosis as demonstrated in a prior study (74). However, there is stronger evidence in the benefit of DCB angioplasty compared to cutting balloon angioplasty with respect to AVF patency (75).

Stent placement in the setting of thrombosed AV access can be considered in certain circumstances to maintain patency of the AV circuit, including elastic recoil after angioplasty, recurrent stenoses, and vessel rupture or pseudoaneurysm resulting in hematoma (25). Stent graft placement in AVG outflow stenosis had been shown to have improved 12-month primary patency compared to angioplasty alone (76). Haskal et al. (66) reported outcomes related to treatment of venous anastomotic stenosis in failing (non-thrombotic) AVG with expanded polytetrafluoroethylene (ePTFE) stent graft. Compared to angioplasty alone, the stenting group had higher 6-month AV circuit patency rate and lower restenosis rate. When evaluating a dialysis patient for stenting, one needs to consider implications in the loss of useable venous segments for future access creation. There are several stent grafts in the market commonly used for dialysis AV access. The Viabahn self-expanding stent graft (W. L. Gore \& Associates; Newark, DE, USA) combines a nitinol exterior with ePTFE lining. The Fluency stent graft (Bard Peripheral Vascular; Tempe, AZ, USA) is also constructed with ePTFE lining within a Nitinol framework. Other available stent grafts include the Wallgraft (Boston Scientific; Natick, MA, USA) a self-expanding stent graft with polyethylene coating, and iCAST (Atrium; Hudson, NH, USA) and the Viabahn VBX, which are balloon expandable ePTFE-coated stents.

\section{Complications}

\section{Vessel rupture and pseudoaneurysm}

Pseudoaneurysm (false aneurysms) and vessel rupture in hemodialysis access can be caused by several factors.
Routine cannulation of $\mathrm{AV}$ access, especially repeated in the same location, can lead to breakdown of native venous or graft wall resulting in pseudoaneurysms $(77,78)$. Increased pressures in AV circuit secondary to outflow stenosis can also predispose the vessel to rupturing (79). In addition, aggressive angioplasty and device manipulation during dialysis access maintenance procedures may lead to injury and subsequent rupture of native venous or graft wall (Figure 5). The reported incidence of vessel or graft rupture related to angioplasty of the AV circuit is $1-16 \%(44,80,81)$. Risk of rupture may be higher when intervention is performed on a recently created AVF or AVG anastomosis.

Rupture or pseudoaneurysm can be treated with prolonged balloon inflation for 2 to 3 minutes for tamponade effect (82). If persistent contrast extravasation occurs despite prolonged balloon inflation, stent grafts may be utilized (83). Stent grafts are lined with ePTFE and can be used to exclude the leak by sealing the neck of the pseudoaneurysm. Studies have demonstrated success in endovascular pseudoaneurysm repair with stent grafts and minimal delay in post-procedure dialysis sessions $(84,85)$. Lastly, care should be exercised during angioplasty of central venous stenoses (e.g., brachiocephalic vein, superior vena cava), as rupture in this location can lead to fatal cardiac tamponade. Kim et al. (86) described an incidence of $0.39 \%$ of central vein rupture during angioplasty of central stenoses in hemodialysis access patients; these ruptures were treated with either balloon tamponade or stenting.

\section{Arterial embolization}

Embolization of thrombi into the arterial system during thrombectomy of clotted $\mathrm{AV}$ access is uncommon and has a reported incidence of $0-7 \%(31,34,37)$. Arterial embolism into the arm may be detected on angiogram or based on signs and symptoms, including pain, paresthesia, pallor, pulselessness, pallor and paralysis. After thrombectomy, it is important to perform an arterial angiogram of the arm and hand especially if there is suspicion of arterial embolism (Figure 6).

The risk of arterial embolism may be reduced by taking the following measures. First, we recommend gentle catheter and wire manipulation when initially navigating past the AV anastomosis into the upstream feeding artery, especially when the clot burden is still high. Second, gentle advancement of the Forgarty thrombectomy balloon into the upstream artery across the AV anastomosis should be practiced when removing the arterial plug. Third, 

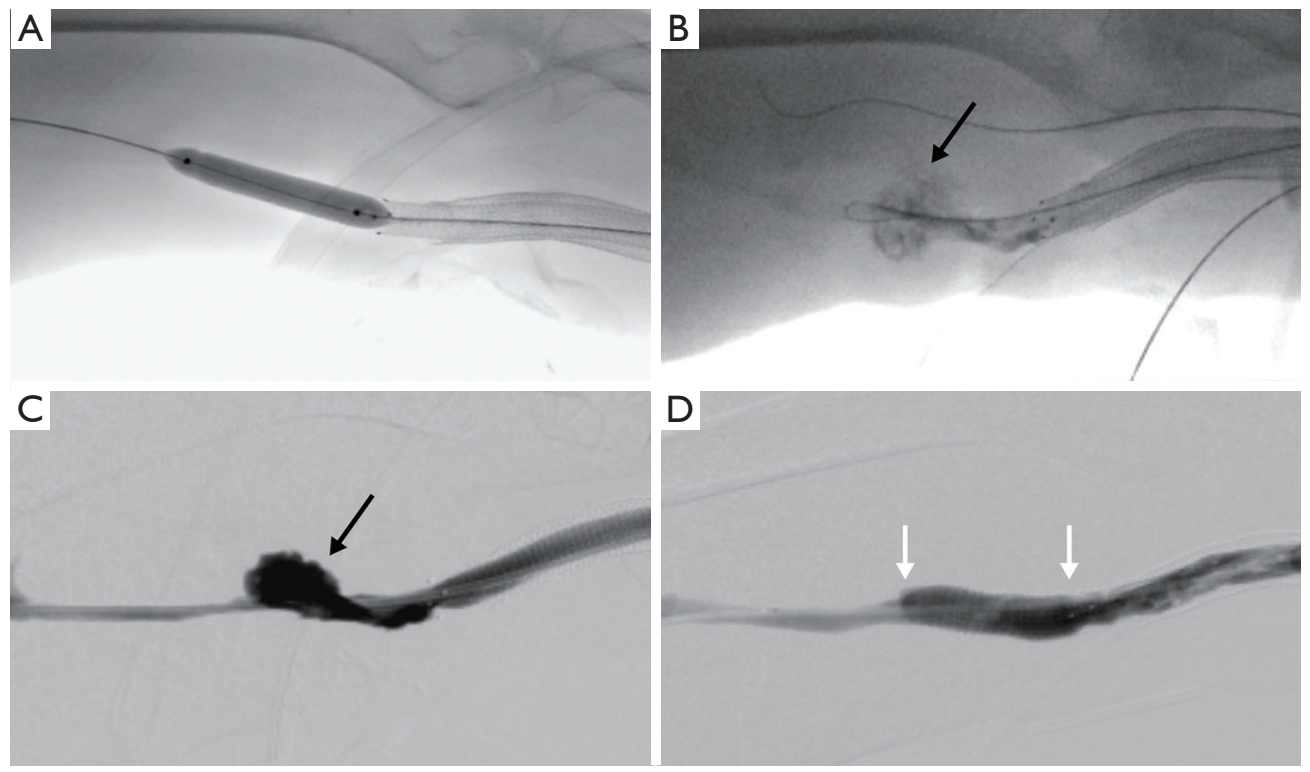

Figure 5 Venous rupture during AVF thrombectomy. (A) Stenosis near the edge of a previously placed venous outflow stent was treated with balloon angioplasty during thrombectomy of a clotted AVF. (B) Venogram shows contrast extravasation at the angioplasty site compatible with venous rupture (black arrow). (C) Repeat venogram after balloon tamponade still showed contained contrast extravasation compatible with a pseudoaneurysm (black arrow). (D) A stent graft was placed (white arrows) to exclude the pseudoaneurysm. AVF, arteriovenous fistula.
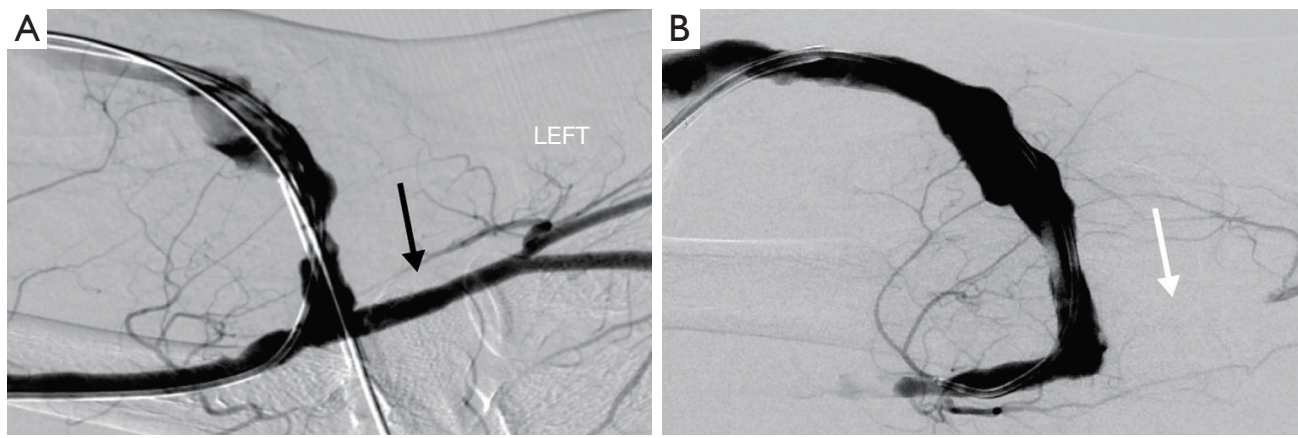

Figure 6 Arterial embolism during AVF thrombectomy. (A) Initial fistulogram with retrograde diagnostic catheter in the upstream brachial artery shows patent downstream brachial artery (black arrow). (B) Repeat fistulogram shows occlusion of the downstream brachial artery (white arrow) concerning for arterial embolus. The thrombus was partially removed using suction thrombectomy followed by systemic anticoagulation. AVF, arteriovenous fistula.

before angioplasty of any arterial inflow stenosis (e.g., juxta-anastomotic segment of AVF or arterial limb of AVG), we recommend removing most of the clot from the circuit first. This would reduce the risk of the balloon inadvertently pushing clot into the artery during angioplasty.

Several techniques have been described to remove the clot that embolized into the upper extremity artery. First, a Fogarty balloon catheter may be advanced from the $\mathrm{AV}$ access into the artery in a retrograde direction. Once the Fogarty catheter is downstream of the thrombus, it is inflated and then pulled back over a wire back into the AV access (87). Next, the Indigo thrombectomy system can be used to remove the arterial embolus. Once the suction catheter is advanced to the location of the clot, the vacuum is turned on and with continuous suction, the catheter is 
retracted back into the AV access sheath. Third, Angiojet can be used to remove arterial clot. However, Angiojet is known to cause fragmentation of thrombi before evacuating the fragments into the catheter, thereby increasing the risk of distal emboli into the hand. Lastly, Trerotola et al. (88) described a back-bleeding method where the proximal feeding artery is occluded with a balloon, resulting in reversal of flow distal to the embolized artery. This will cause the embolus to move toward $\mathrm{AV}$ access and away from the artery.

\section{$P E$}

Clinically significant PE following thrombectomy of clotted hemodialysis access is uncommon. Swan et al. (89) reported that $\mathrm{PE}$ was detected on perfusion scan in 59\% of the patients following AVG declot, but only $5 \%$ of these patients with positive PE studies were symptomatic. Another study showed that PE was detected on perfusion scan in $70 \%$ of the cases after PMT of clotted AVG. However all of the PE's were asymptomatic (90). Routine periprocedural systemic heparin may decrease the impact of PE. Aspiration thrombectomy using the vascular sheath (with manual aspiration), Indigo thrombectomy system, or Angiojet may be considered to reduce the amount of clot that embolizes in the pulmonary artery. In patients with low pulmonary reserve who require thrombectomy of their clotted AV access, a careful discussion of the risk and benefits of the procedure is recommended.

\section{Paradoxical embolism}

Paradoxical embolism to the brain occurs in patients with intracardiac right-to-left shunts who develop deep venous thrombosis and PE. During thrombectomy of AV access, clot fragments are invariably dislodged and embolized into the pulmonary arterial system (91). Moreover, patients with patent foramen ovale (PFO) are at risk for paradoxical embolism into the arterial circulation, especially in those with increased right heart pressures related to chronic lung disease, chronic thromboembolic pulmonary hypertension, and Valsalva maneuver (92). A previous retrospective study had shown safety in AV access declot in 23 patients with known PFO (93). Nonetheless, careful discussion of risk and benefits should be done when evaluating for declot procedures in hemodialysis patients with known right-toleft shunts or PFO.

\section{Surgical thrombectomy}

Surgery is an established technique to salvage a thrombosed $\mathrm{AV}$ access. The surgical technique involves the use of a Fogarty thrombectomy catheter with retrograde manual clot removal. The advantages of surgery thrombectomy include lower risks of thrombus dislocation, minimal use of thrombolytics, and the ability to surgically repair ruptured access or pseudoaneurysm during the same operation. In large aneurysmal fistulas, surgical thrombectomy is preferred for evacuation of chronic wall-adherent thrombus, and the aneurysm can be revised at the same time. Technical success rates for surgical thrombectomy of $\mathrm{AV}$ access are $70-94 \%$ with 12 -month patency rates of $68-88 \%$ for either AVF or AVG $(17,94,95)$.

A recent meta-analysis comparing outcomes between endovascular and surgical thrombectomy in AVG thrombosis (24) concluded that no significant differences in primary patency rates were observed between the two techniques under 90 days. Additionally, no differences in complication rates were observed between the two groups. However, endovascular thrombectomy had inferior long term patency rates at first and second year compared to surgery. Endovascular thrombectomy was also associated with higher technical failure rates compared to its surgical counterpart.

\section{Conclusions}

Thrombectomy of clotted AVF or AVG prevents missed or delayed hemodialysis sessions and abandonment of access. Access surveillance may detect issues with the AV circuit early before thrombosis. Endovascular techniques have largely replaced surgery in the treatment of clotted access. Although thrombectomy techniques may vary depending on the operator and device availability, they all include the general steps of clot removal and dilatation of underlying stenosis that precipitated the thrombosis. Recent studies have shown that most thrombectomy procedures involve a variation of PMT with and without advanced thrombectomy devices, such as rheolytic or vacuum-assisted thrombectomy. Adjunctive treatments of refractory stenosis in the circuit using DCB or stent graft may improve long term patency of $\mathrm{AV}$ access. Thrombectomy of clotted AV access is generally safe with high technical and clinical success rates. Patient selection is important in preventing major complications such as clinically significant $\mathrm{PE}$ and paradoxical embolism. 
Complications specific to thrombectomy such as vessel/ graft rupture and arterial embolism can be prevented or treated if they do occur.

\section{Acknowledgments}

Funding: None.

\section{Footnote}

Provenance and Peer Review: This article was commissioned by the Guest Editors (Sasan Partovi and Lee Kirksey) for the series "Endovascular and Surgical Interventions in the End Stage Renal Disease Population" published in Cardiovascular Diagnosis and Therapy. The article has undergone external peer review.

Reporting Checklist: The authors have completed the Narrative Review reporting checklist. Available at https:// cdt.amegroups.com/article/view/10.21037/cdt-21-523/rc

Conflicts of Interest: All authors have completed the ICMJE uniform disclosure form (available at https://cdt.amegroups. com/article/view/10.21037/cdt-21-523/coif). The series "Endovascular and Surgical Interventions in the End Stage Renal Disease Population" was commissioned by the editorial office without any funding or sponsorship. SPK serves as an unpaid editorial board member of Cardiovascular Diagnosis and Therapy from September 2021 to August 2023. SPK reports personal fees from Elsevier, Springer, Koo Foundation, Medtronic, Penumbra, US Vascular, and Dova pharmaceuticals. SPK is an investor of AltheaHealth. The authors have no other conflicts of interest to declare.

Ethical Statement: The authors are accountable for all aspects of the work in ensuring that questions related to the accuracy or integrity of any part of the work are appropriately investigated and resolved.

Open Access Statement: This is an Open Access article distributed in accordance with the Creative Commons Attribution-NonCommercial-NoDerivs 4.0 International License (CC BY-NC-ND 4.0), which permits the noncommercial replication and distribution of the article with the strict proviso that no changes or edits are made and the original work is properly cited (including links to both the formal publication through the relevant DOI and the license). See: https://creativecommons.org/licenses/by-nc-nd/4.0/.

\section{References}

1. Dhingra RK, Young EW, Hulbert-Shearon TE, et al. Type of vascular access and mortality in U.S. hemodialysis patients. Kidney Int 2001;60:1443-51.

2. Lacson E Jr, Wang W, Lazarus JM, et al. Change in vascular access and hospitalization risk in longterm hemodialysis patients. Clin J Am Soc Nephrol 2010;5:1996-2003.

3. Quencer KB, Friedman T. Declotting the Thrombosed Access. Tech Vasc Interv Radiol 2017;20:38-47.

4. Al-Jaishi AA, Liu AR, Lok CE, et al. Complications of the Arteriovenous Fistula: A Systematic Review. J Am Soc Nephrol 2017;28:1839-50.

5. Salman L, Rizvi A, Contreras G, et al. A Multicenter Randomized Clinical Trial of Hemodialysis Access Blood Flow Surveillance Compared to Standard of Care: The Hemodialysis Access Surveillance Evaluation (HASE) Study. Kidney Int Rep 2020;5:1937-44.

6. Knoll GA, Wells PS, Young D, et al. Thrombophilia and the risk for hemodialysis vascular access thrombosis. J Am Soc Nephrol 2005;16:1108-14.

7. Salmela B, Hartman J, Peltonen S, et al. Thrombophilia and arteriovenous fistula survival in ESRD. Clin J Am Soc Nephrol 2013;8:962-8.

8. Maoz D, Reinitz R, Rimon U, et al. Hemodialysis graft flow surveillance with prompt corrective interventions improves access long-term patency. Clin Nephrol 2009;71:43-9.

9. Besarab A, Bolton WK, Browne JK, et al. The effects of normal as compared with low hematocrit values in patients with cardiac disease who are receiving hemodialysis and epoetin. N Engl J Med 1998;339:584-90.

10. Lee T, Barker J, Allon M. Needle infiltration of arteriovenous fistulae in hemodialysis: risk factors and consequences. Am J Kidney Dis 2006;47:1020-6.

11. Shah A, Ansari N, Hamadeh Z. Cardiac Arrest Secondary to Bilateral Pulmonary Emboli following Arteriovenous Fistula Thrombectomy: A Case Report with Review of the Literature. Case Rep Nephrol 2012;2012:831726.

12. Crowther MA, Clase CM, Margetts PJ, et al. Lowintensity warfarin is ineffective for the prevention of PTFE graft failure in patients on hemodialysis: a randomized controlled trial. J Am Soc Nephrol 2002;13:2331-7.

13. Palmer SC, Di Micco L, Razavian M, et al. Antiplatelet therapy to prevent hemodialysis vascular access failure: systematic review and meta-analysis. Am J Kidney Dis 2013;61:112-22. 
14. Hsieh MY, Lin L, Chen TY, et al. Timely thrombectomy can improve patency of hemodialysis arteriovenous fistulas. J Vasc Surg 2018;67:1217-26.

15. Drouven JW, de Bruin C, van Roon AM, et al. Outcomes after endovascular mechanical thrombectomy in occluded vascular access used for dialysis purposes. Catheter Cardiovasc Interv 2020;95:758-64.

16. Schon D, Mishler R. Pharmacomechanical thrombolysis of natural vein fistulas: reduced dose of TPA and long-term follow-up. Semin Dial 2003;16:272-5.

17. Lipari G, Tessitore N, Poli A, et al. Outcomes of surgical revision of stenosed and thrombosed forearm arteriovenous fistulae for haemodialysis. Nephrol Dial Transplant 2007;22:2605-12.

18. Kakkos SK, Haddad GK, Haddad JA, et al. Secondary patency of thrombosed prosthetic vascular access grafts with aggressive surveillance, monitoring and endovascular management. Eur J Vasc Endovasc Surg 2008;36:356-65.

19. United States Renal Data System. 2019 USRDS annual data report: Epidemiology of kidney disease in the United States. National Institutes of Health, National Institute of Diabetes and Digestive and Kidney Diseases, 2019. Available online: https://www.usrds.org/annual-data-report/

20. Ram SJ, Nassar R, Work J, et al. Risk of hemodialysis graft thrombosis: analysis of monthly flow surveillance. Am J Kidney Dis 2008;52:930-8.

21. Allon M, Robbin ML. Hemodialysis vascular access monitoring: current concepts. Hemodial Int 2009; 13:153-62.

22. MacRae JM, Dipchand C, Oliver M, et al. Arteriovenous Access Failure, Stenosis, and Thrombosis. Can J Kidney Health Dis 2016;3:2054358116669126.

23. Vesely TM. Endovascular intervention for the failing vascular access. Adv Ren Replace Ther 2002;9:99-108.

24. Chan N, Wee I, Soong TK, et al. A systematic review and meta-analysis of surgical versus endovascular thrombectomy of thrombosed arteriovenous grafts in hemodialysis patients. J Vasc Surg 2019;69:1976-1988.e7.

25. Dariushnia SR, Walker TG, Silberzweig JE, et al. Quality Improvement Guidelines for Percutaneous Image-Guided Management of the Thrombosed or Dysfunctional Dialysis Circuit. J Vasc Interv Radiol 2016;27:1518-30.

26. Turmel-Rodrigues L, Pengloan J, Rodrigue H, et al. Treatment of failed native arteriovenous fistulae for hemodialysis by interventional radiology. Kidney Int 2000;57:1124-40.

27. Turmel-Rodrigues L, Raynaud A, Louail B, et al. Manual catheter-directed aspiration and other thrombectomy techniques for declotting native fistulas for hemodialysis. J Vasc Interv Radiol 2001;12:1365-71.

28. Hunter DW, Castaneda-Zuniga WR, Coleman CC, et al. Failing arteriovenous dialysis fistulas: evaluation and treatment. Radiology 1984;152:631-5.

29. Zeit RM, Cope C. Failed hemodialysis shunts. One year of experience with aggressive treatment. Radiology 1985;154:353-6.

30. Poulain F, Raynaud A, Bourquelot P, et al. Local thrombolysis and thromboaspiration in the treatment of acutely thrombosed arteriovenous hemodialysis fistulas. Cardiovasc Intervent Radiol 1991;14:98-101.

31. Summers S, Drazan K, Gomes A, et al. Urokinase therapy for thrombosed hemodialysis access grafts. Surg Gynecol Obstet 1993;176:534-8.

32. Schuman E, Quinn S, Standage B, et al. Thrombolysis versus thrombectomy for occluded hemodyalisis grafts. Am J Surg 1994;167:473-6.

33. Cohen MA, Kumpe DA, Durham JD, et al. Improved treatment of thrombosed hemodialysis access sites with thrombolysis and angioplasty. Kidney Int 1994;46:1375-80.

34. Valji K. Transcatheter treatment of thrombosed hemodialysis access grafts. AJR Am J Roentgenol 1995;164:823-9.

35. Davis GB, Dowd CF, Bookstein JJ, et al. Thrombosed dialysis grafts: efficacy of intrathrombic deposition of concentrated urokinase, clot maceration, and angioplasty. AJR Am J Roentgenol 1987;149:177-81.

36. Wildberger J, Schmitz-Rode T, Wein BB, et al. Mechanical thrombectomy in hemodialysis access shunts using a $5 \mathrm{~F}$ pigtail rotation catheter. In vitro and in vivo investigations. Invest Radiol 1999;34:489-95.

37. Sands JJ, Patel S, Plaviak DJ, et al. Pharmacomechanical thrombolysis with urokinase for treatment of thrombosed hemodialysis access grafts. A comparison with surgical thrombectomy. ASAIO J 1994;40:M886-8.

38. Cynamon J, Lakritz PS, Wahl SI, et al. Hemodialysis graft declotting: description of the "lyse and wait" technique. J Vasc Interv Radiol 1997;8:825-9.

39. Bookstein JJ, Fellmeth B, Roberts A, et al. Pulsed-spray pharmacomechanical thrombolysis: preliminary clinical results. AJR Am J Roentgenol 1989;152:1097-100.

40. Valji K, Bookstein JJ, Roberts AC, et al. Pharmacomechanical thrombolysis and angioplasty in the management of clotted hemodialysis grafts: early and late clinical results. Radiology 1991;178:243-7.

41. Valji K, Bookstein JJ, Roberts AC, et al. Pulse-spray pharmacomechanical thrombolysis of thrombosed 
hemodialysis access grafts: long-term experience and comparison of original and current techniques. AJR Am J Roentgenol 1995;164:1495-500; discussion 1501-3.

42. Beathard GA. Mechanical versus pharmacomechanical thrombolysis for the treatment of thrombosed dialysis access grafts. Kidney Int 1994;45:1401-6.

43. Cooper SG. Original report. Pulse-spray thrombolysis of thrombosed hemodialysis grafts with tissue plasminogen activator. AJR Am J Roentgenol 2003;180:1063-6.

44. Choi SY, Choi BG, Han KH, et al. Efficacy of a modified pharmacomechanical thrombolysis technique for endovascular treatment of thrombosed prosthetic arteriovenous grafts. Korean J Radiol 2012;13:300-6.

45. Winkler TA, Trerotola SO, Davidson DD, et al. Study of thrombus from thrombosed hemodialysis access grafts. Radiology 1995;197:461-5.

46. Nassar GM, Rhee E, Khan AJ, et al. Percutaneous thrombectomy of AVF: immediate success and long-term patency rates. Semin Dial 2015;28:E15-22.

47. Zaleski GX, Funaki B, Gentile L, et al. Purse-string sutures and miniature tourniquet to achieve immediate hemostasis of percutaneous grafts and fistulas: a simple trick with a twist. AJR Am J Roentgenol 2000;175:1643-5.

48. Rocek M, Peregrin JH, Lasovicková J, et al. Mechanical thrombolysis of thrombosed hemodialysis native fistulas with use of the Arrow-Trerotola percutaneous thrombolytic device: our preliminary experience. J Vasc Interv Radiol 2000;11:1153-8.

49. Trerotola SO, Vesely TM, Lund GB, et al. Treatment of thrombosed hemodialysis access grafts: ArrowTrerotola percutaneous thrombolytic device versus pulsespray thrombolysis. Arrow-Trerotola Percutaneous Thrombolytic Device Clinical Trial. Radiology 1998;206:403-14.

50. Vashchenko N, Korzets A, Neiman C, et al. Retrospective comparison of mechanical percutaneous thrombectomy of hemodialysis arteriovenous grafts with the ArrowTrerotola device and the lyse and wait technique. AJR Am J Roentgenol 2010;194:1626-9.

51. Huan KWSJ, Tan CS, Chua D, et al. The Cleaner $\mathrm{XT}^{\mathrm{TM}}$ Device as an Endovascular Adjunct for Pharmacomechanical Thrombolysis of Thrombosed Arteriovenous Fistulas and Grafts. Ann Vasc Dis 2020;13:390-6.

52. Webb AH, Zacharias K, Ciowlek P, et al. Utility of Rotational Thrombectomy for the Management of Thrombosed Arteriovenous Shunts. The Arab Journal of Interventional Radiology 2020;4:S7.
53. Yasui K, Qian Z, Nazarian GK, et al. Recirculation-type Amplatz clot macerator: determination of particle size and distribution. J Vasc Interv Radiol 1993;4:275-8.

54. Haage P, Vorwerk D, Wildberger JE, et al. Percutaneous treatment of thrombosed primary arteriovenous hemodialysis access fistulae. Kidney Int 2000;57:1169-75.

55. Dwarka D, Schwartz SA, Smyth SH, et al. Bradyarrhythmias during use of the AngioJet system. J Vasc Interv Radiol 2006;17:1693-5.

56. Littler P, Cullen N, Gould D, et al. AngioJet thrombectomy for occluded dialysis fistulae: outcome data. Cardiovasc Intervent Radiol 2009;32:265-70.

57. Simoni E, Blitz L, Lookstein R. Outcomes of AngioJet ${ }^{\circledR}$ thrombectomy in hemodialysis vascular access grafts and fistulas: PEARL I Registry. J Vasc Access 2013;14:72-6.

58. Bermudez P, Fontseré N, Mestres G, et al. Endovascular Revascularization of Hemodialysis Thrombosed Grafts with the Hydrodynamic Thrombectomy Catheter. Our 7-Year Experience. Cardiovasc Intervent Radiol 2017;40:252-9.

59. Lee HY, Kim CS, Han KD, et al. Comparison of the Clinical Outcomes between Angiojet Pharmacomechanical Thrombectomy and Hybrid Surgical Thrombectomy for Thrombotic Occlusion of Hemodialysis Access. Vasc Specialist Int 2020;36:241-7.

60. Aydın E, Bademci MŞ, Kocaaslan C. Comparison of pharmacomechanical and surgical interventions for thrombosed native arteriovenous fistulas. Turk Gogus Kalp Damar Cerrahisi Derg 2020;28:609-14.

61. Owens CA, Yaghmai B, Aletich V, et al. Fatal paradoxic embolism during percutaneous thrombolysis of a hemodialysis graft. AJR Am J Roentgenol 1998;170:742-4.

62. Marcelin C, D'Souza S, Le Bras Y, et al. Mechanical Thrombectomy in Acute Thrombosis of Dialysis Arteriovenous Fistulae and Grafts Using a VacuumAssisted Thrombectomy Catheter: A Multicenter Study. J Vasc Interv Radiol 2018;29:993-7.

63. Piacentino F, Coppola A, Zaghetto A, et al. Vacuumassisted mechanical thrombectomy in extensively occlusive thrombosis of dialysis arteriovenous grafts with indigo system. J Vasc Access 2020;21:673-9.

64. Roy-Chaudhury P, Sukhatme VP, Cheung AK. Hemodialysis vascular access dysfunction: a cellular and molecular viewpoint. J Am Soc Nephrol 2006;17:1112-27.

65. Lee T, Roy-Chaudhury P. Advances and new frontiers in the pathophysiology of venous neointimal hyperplasia and dialysis access stenosis. Adv Chronic Kidney Dis 2009;16:329-38. 
66. Haskal ZJ, Trerotola S, Dolmatch B, et al. Stent graft versus balloon angioplasty for failing dialysis-access grafts. N Engl J Med 2010;362:494-503.

67. Irani FG, Teo TKB, Tay KH, et al. Hemodialysis Arteriovenous Fistula and Graft Stenoses: Randomized Trial Comparing Drug-eluting Balloon Angioplasty with Conventional Angioplasty. Radiology 2018;289:238-47.

68. Swinnen JJ, Hitos K, Kairaitis L, et al. Multicentre, randomised, blinded, control trial of drug-eluting balloon vs Sham in recurrent native dialysis fistula stenoses. J Vasc Access 2019;20:260-9.

69. Trerotola SO, Lawson J, Roy-Chaudhury P, et al. Drug Coated Balloon Angioplasty in Failing AV Fistulas: A Randomized Controlled Trial. Clin J Am Soc Nephrol 2018;13:1215-24.

70. Trerotola SO, Saad TF, Roy-Chaudhury P, et al. The Lutonix AV Randomized Trial of Paclitaxel-Coated Balloons in Arteriovenous Fistula Stenosis: 2-Year Results and Subgroup Analysis. J Vasc Interv Radiol 2020;31:1-14.e5.

71. Lookstein RA, Haruguchi H, Ouriel K, et al. Drug-Coated Balloons for Dysfunctional Dialysis Arteriovenous Fistulas. N Engl J Med 2020;383:733-42.

72. Liao MT, Lee CP, Lin TT, et al. A randomized controlled trial of drug-coated balloon angioplasty in venous anastomotic stenosis of dialysis arteriovenous grafts. J Vasc Surg 2020;71:1994-2003.

73. Tan CW, Tan RY, Pang SC, et al. Single-Center Prospective Pilot Study of Sirolimus Drug-Coated Balloon Angioplasty in Maintaining the Patency of Thrombosed Arteriovenous Graft. J Vasc Interv Radiol 2021;32:369-75.

74. Agarwal SK, Nadkarni GN, Yacoub R, et al. Comparison of Cutting Balloon Angioplasty and Percutaneous Balloon Angioplasty of Arteriovenous Fistula Stenosis: A MetaAnalysis and Systematic Review of Randomized Clinical Trials. J Interv Cardiol 2015;28:288-95.

75. Tripsianis G, Christaina E, Argyriou C, et al. Network meta-analysis of trials comparing first line endovascular treatments for arteriovenous fistula stenosis. J Vasc Surg 2021;73:2198-2203.e3.

76. Carmona J, Rits Y, Jones B, et al. Patency of the Viabahn stent graft for the treatment of outflow stenosis in hemodialysis grafts. Am J Surg 2016;211:551-4.

77. Vesely TM. Use of stent grafts to repair hemodialysis graft-related pseudoaneurysms. J Vasc Interv Radiol 2005;16:1301-7.

78. Moszkowicz A, Behrens G, Gueyikian S, et al. Occlusion of a rapidly expanding hemodialysis graft pseudoaneurysm with placement of a stent graft. Semin Intervent Radiol 2007;24:34-7.

79. Ryan JM, Dumbleton SA, Doherty J, et al. Technical innovation. Using a covered stent (wallgraft) to treat pseudoaneurysms of dialysis grafts and fistulas. AJR Am J Roentgenol 2003;180:1067-71.

80. Welber A, Schur I, Sofocleous CT, et al. Endovascular stent placement for angioplasty-induced venous rupture related to the treatment of hemodialysis grafts. J Vasc Interv Radiol 1999;10:547-51.

81. Lee CK, Hsieh MC, Luo CM, et al. Use of the Viabahn covered stent for the treatment of venous rupture during interventions of dysfunctional or thrombosed hemodialysis vascular access. J Vasc Access 2021;22:759-66.

82. Kornfield ZN, Kwak A, Soulen MC, et al. Incidence and management of percutaneous transluminal angioplastyinduced venous rupture in the "fistula first" era. J Vasc Interv Radiol 2009;20:744-51.

83. Bent CL, Rajan DK, Tan K, et al. Effectiveness of stent-graft placement for salvage of dysfunctional arteriovenous hemodialysis fistulas. J Vasc Interv Radiol 2010;21:496-502.

84. Barshes NR, Annambhotla S, Bechara C, et al.

Endovascular repair of hemodialysis graft-related pseudoaneurysm: an alternative treatment strategy in salvaging failing dialysis access. Vasc Endovascular Surg 2008;42:228-34.

85. Najibi S, Bush RL, Terramani TT, et al. Covered stent exclusion of dialysis access pseudoaneurysms. J Surg Res 2002;106:15-9.

86. Kim YJ, Yang SB, Lee WH, et al. Central vein rupture during percutaneous transluminal angioplasty for central vein stenosis or occlusion in haemodialysis patients. S Afr J $\operatorname{Rad} 2017 ; 21: a 1205$.

87. Goo DE, Yang SB, Kim YJ, et al. Arterial Embolism Occurring During Percutaneous Thrombectomy of Dialysis Graft. Cardiovasc Intervent Radiol 2017;40:1866-72.

88. Trerotola SO, Johnson MS, Shah H, et al. Backbleeding technique for treatment of arterial emboli resulting from dialysis graft thrombolysis. J Vasc Interv Radiol 1998;9:141-3.

89. Swan TL, Smyth SH, Ruffenach SJ, et al. Pulmonary embolism following hemodialysis access thrombolysis/ thrombectomy. J Vasc Interv Radiol 1995;6:683-6.

90. Kinney TB, Valji K, Rose SC, et al. Pulmonary embolism from pulse-spray pharmacomechanical thrombolysis of clotted hemodialysis grafts: urokinase versus heparinized 
saline. J Vasc Interv Radiol 2000;11:1143-52.

91. Bentaarit B, Duval AM, Maraval A, et al. Paradoxical embolism following thromboaspiration of an arteriovenous fistula thrombosis: a case report. J Med Case Rep 2010;4:345.

92. Santos JP, Hamadeh Z, Ansari N. Cerebrovascular accident secondary to paradoxical embolism following arteriovenous graft thrombectomy. Case Rep Nephrol 2012;2012:183730.

93. Wu S, Ahmad I, Qayyum S, et al. Paradoxical embolism

Cite this article as: $\mathrm{Wu} \mathrm{V}$, Kalva SP, Cui J. Thrombectomy approach for access maintenance in the end stage renal disease population: a narrative review. Cardiovasc Diagn Ther 2023;13(1):265-280. doi: 10.21037/cdt-21-523 after declotting of hemodialysis fistulae/grafts in patients with patent foramen ovale. Clin J Am Soc Nephrol 2011;6:1333-6.

94. Ponikvar R, Premru V, Kersnič B. Surgical thrombectomy of thrombosed arteriovenous grafts by interventional nephrologists. Ther Apher Dial 2011;15:306-10.

95. Ponikvar R. Surgical salvage of thrombosed native arteriovenous fistulas for hemodialysis by interventional nephrologists. Ther Apher Dial 2009;13:340-4. 